\title{
A green method to prepare nanosilica modified graphene oxide to inhibit nanoparticles re-aggregation during melt processing
}

\author{
Roberto Scaffaro*, Andrea Maio \\ Department of Civil, Environmental, Aerospace, Materials Engineering, University of Palermo, Viale delle Scienze, Ed. 6, 90128 Palermo, Italy
}

\section{A R T I C L E I N F O}

Article history:

Received 29 June 2016

Received in revised form 8 September 2016

Accepted 28 September 2016

Available online xxx

Keywords:

Green processing

SEM

Dynamic mechanical thermal analysis (DMTA)

Mechanical properties

Interphase

Graphene-ceramics
A B S T R A C T

A graphene oxide-silica nanohybrid (GOS), self-assembled into a lasagna-like structure, was prepared in water and used as a filler for the melt preparation of polyamide 6 (PA6)-based nanocomposites. For sake of comparison, PA6-based materials were prepared under the same processing conditions by adding GO only or a physical mixture of GO and silica $(\mathrm{GO}+\mathrm{S})$. All the materials were characterized from a morphological, spectroscopic, thermal, dynamic-mechanical (DMA) and mechanical point of view. For all the nanocomposites, the interphase was studied either by analyzing loss factor plots coming from DMA measurements and by implementing a novel approach, i.e. combining solvent extraction, SEM and EDX measurements. While GO and GO+S showed an almost negligible effect on the macroscopic features of PA6, due to lack of dispersion, PA6-GOS ternary nanocomposites displayed an outstanding enhancement of mechanical and thermo-mechanical performance. This feature is likely due to the formation of an extended and strong interphase. In fact, the silica layers intercalated between GO lamellae played as an exfoliating agent, thus proving to be particularly efficient in avoiding self-aggregation of GO sheets, while those covalently attached to basal planes of GO acted as a rivet, capable to interlock the surrounding polymer chains. Finally, with respect to other techniques commonly adopted for the fabrication of polymer-graphene nanocomposites, the green route herein proposed does not involve any toxic solvent nor time-consuming protocols, and allows achieving remarkable improvements in stiffening (up to $+180 \%$ ), strengthening (up to $+210 \%$ ) and toughening (up to $+210 \%$ ) at extremely low filler contents $(0.25 \%$ or $0.5 \%$ ).

(C) 2016 Published by Elsevier Ltd

\section{Introduction}

Graphene oxide $(\mathrm{GO})$ can be considered as a graphene layer modified with oxygen functional groups decorating the basal planes and edges. Its dual honeycomb, constituted by both $\mathrm{sp}^{2}$ and $\mathrm{sp}^{3}$ domains, as well as the presence of a variegated assortment of functional moieties, enable the attainment of unique and intriguing properties from a chemical-physical point of view [1,2]. Hence, the incorporation of GO to polymers offers significant potential for the development of advanced materials in several applications [3]. In this context, polyamide 6 (PA6)-GO systems were widespreadly investigated, aiming at the possibility to improve the dimensional stability and mechanical performance of PA6 [4].

However, despite its good mechanical properties and its potential affinity to PA6, GO lamellae present several drawbacks in terms of dispersion and processability $[4,5]$. When GO is melt processed together with polymers, in fact, pleating and crumpling phenomena occur due to several reasons, such as the thermally-driven formation of intramolecular and intermolecular reactions occurring between oxygen moieties of GO, the mechanical cleavage or distortion of lamellae under shear stress, promoted by the presence of several structural defects, the volatilization of the water integrated within GO structure [5].

* Corresponding author.

Email address: roberto.scaffaro@unipa.it (R. Scaffaro)
Up to date, several strategies have been adopted to prepare PA6-GO nanocomposites with adequate levels of dispersion [3-5]. Basically, all of these approaches can be classified into three categories, each of them presenting their own positive and negative features:

(i) in situ polymerization;

(ii) solvent blending;

(iii) multi-step routes combining (i) or (ii) and/or GO derivatization prior to melt processing.

In situ polymerization is the most commonly used preparative, as it provides good dispersion of GO in polymer matrix and strong interfacial adhesion, although several issues currently limit its industrial scalability, such as the increase of viscosity that hinders manipulation and loading contents higher than $0.1 \%$, the use of chemicals for the derivatization that make it environment unfriendly, time-consuming reactions $[3,4]$.

On the other hand, solvent blending provides a simple route to incorporate $\mathrm{GO}$ into polymer matrices, achieving high degree of dispersion but, conversely, the use of surfactants may often affect polymer properties and pose serious issues in terms of solvent removal and environmental risks [4,5]. Recently, many efforts have been devoted to the development of new solvents, capable to enhance the properties of polyamide-graphene nanocomposites [6].

The main limitations of these techniques refer to the scarce industrial scalability, essentially due to the lack of cost-effectiveness, the use of toxic reagents and time-consuming processes [3-5,7-10].

In order to limit the disadvantages related to aforementioned procedures, some papers report on the use of two-step procedures, in- 
volving an in situ polymerization or solvent blending step to pre-disperse GO prior to melt processing [5]. We recently demonstrated that a two-step technique, involving the preparation of a masterbatch via wet phase inversion and further melt processing with a polymer, dramatically enhanced the dispersion reducing the solvent amount. However, this approach requires large amounts of water, thus presenting some difficulties out of a lab scale [5]. More generally, pre-dispersing GO in a PA6 needs at least a solvent-based step, since liquid feeding melt extrusion, conveniently adopted for dispersing $\mathrm{GO}$ within poly(vinylidene fluoride) using aqueous GO [11], is hindered in the case of polymers particularly susceptible to hydrolytic scission, such as PA6. In this perspective, a significant goal may be the use of appropriate solid pre-dispersants to inhibit GO re-aggregation during melt processing.

Indeed, we previously reported that the direct exfoliation and conjugation of GO to silica nanoparticles can be carried out in water by a simple hydrothermal treatment requiring less than $30 \mathrm{~min}[12,13]$. Silica nanoparticles are covalently bonded to the GO surface by Fischer esterification exploiting the intrinsic acidity of GO itself in aqueous dispersion. The development of a unique, simple and low-energy synthesis route for the formation of GO-silica nanohybrid (GOS) self-organizing into a lasagna-like assembly, inspired us to use silica nanoparticles as solid-state exfoliants to inhibit the re-aggregation of GO sheets during melt processing.

On the other hand, the possibility to use graphemic compounds decorated with several nanoparticles, such as metal ( $\mathrm{Au}, \mathrm{Ni})$, metal oxides (especially $\mathrm{Fe}_{3} \mathrm{O}_{4}$ and alumine) and ceramics, as nanohybrid fillers for polymeric materials is gaining an increasing interest during the last years owing to the possibility to tune certain characteristics, such as thermal, electrical, magnetic and mechanical performance, or even to equip the nanocomposites with additional properties, useful to develop multifunctional materials [9,14-19].

Among these ternary nanocomposites, particular attention has been recently devoted to polymeric nanocomposites containing both GO and silica [20]. In this latter case, the possibility to combine some peculiar aspects of the silica, such as its high mechanical strength and thermal stability, with the typical flexibility and processability of the polymers, can be promoted by the intrinsic ability of GO to create structured nanointerfaces highly interacting with polymer and silica. To the best of our knowledge, however, the fabrication of a polymer-GO-silica nanocomposite by melt processing, as well as the possibility to use solid exfoliants to inhibit GO re-aggregation, have been not yet reported so far.

Aim of this work is to present a novel, green, simple and cost-effective strategy to directly feed GOS and PA6 into a batch mixer without any further preparation step, thus exploring the possibility to maximize GO dispersion without involving organic solvent and, furthermore, to gather the unique properties of PA6, GO and silica, into an integrated ternary nanocomposite.

\section{Experimental part}

\subsection{Materials and methods}

The PA6 used in the frame of this work was a sample of Radilon S35 100 NAT (density $=1.14 \mathrm{~g} \cdot \mathrm{cm}^{-3}$, viscosity index in sulfuric acid $=205 \mathrm{ml} \cdot \mathrm{g}^{-1}$ ), supplied by Radicinova (Italy). Neat graphite ( maximum lateral size $=45 \mu \mathrm{m}$, density $=2.1 \mathrm{~g} \cdot \mathrm{cm}^{-3}$ ) used as starting material for the synthesis of GO was purchased by NGS Naturgraphit (Germany), silica nanoparticles Aerosil 300 (specific surface $=300$ $\mathrm{m}^{2} \cdot \mathrm{g}^{-1}$, diameter $=7 \mathrm{~nm}$, density $2.2 \mathrm{~g} \cdot \mathrm{cm}^{-3}$ ) were provided by Evonik Industries (Germany).
GO was synthesized via oxidation and subsequent chemical exfoliation of graphite by slightly modifying Tour's method [21], more details about the synthesis are reported elsewhere [22].

GOS nanohybrids were synthesized via Fischer esterification in water. Briefly, GO (0.2 g) and silica nanoparticles $(0.2 \mathrm{~g})$ were dispersed in water $(150 \mathrm{~mL})$ with $2 \% \mathrm{HCOOH}$, sonicated at $50{ }^{\circ} \mathrm{C}$ for $1 \mathrm{~h}$ and then transferred to a Teflon-coated crystallizer, where the dispersion was magnetic-stirred at $120{ }^{\circ} \mathrm{C}$. Of course, as long as the solvent was present, the temperature remained constant at $100{ }^{\circ} \mathrm{C}$. After the total evaporation of water, the gel-like slurry was maintained at $120{ }^{\circ} \mathrm{C}$ for $15 \mathrm{~min}$. Finally, a dark brown film was peeled off and ground into flakes. More details can be found elsewhere [12]

The PA6-GOS nanocomposites were prepared at two different loading levels $(0.25 \%$ or $0.5 \%$ weight percentage $)$. For sake of comparison, PA6 containing either GO $(0.5 \%)$ or a solid mixture of GO $(0.25 \%)$ and silica $(0.25 \%)$ were prepared too. For a typical preparation of nanocomposites, $50 \mathrm{~g}$ of polymer and fillers, conveniently dried in vacuo at $80{ }^{\circ} \mathrm{C}$ overnight, were pre-mixed at the solid state, fed to a Brabender Plasticorder PLE300 batch mixer and then processed for $6 \mathrm{~min}$ at constant rotor speed $(100 \mathrm{rpm})$ and temperature $\left(\mathrm{T}=240{ }^{\circ} \mathrm{C}\right)$. Thereafter, the materials - fed out of the batch mixer - were rapidly cooled, ground into pellets, compression molded for about $2 \mathrm{~min}$ at the same temperature as that used for the processing and finally cut into specimens for further characterizations.

\subsection{Characterization}

Spectroscopic analysis was used to confirm the effective silica-decoration of GO. FT-IR/ATR analysis was carried out by using a Perkin-Elmer FT-IR/NIR Spectrum 400 spectrophotometer, the spectra were recorded in the range $4000-400 \mathrm{~cm}^{-1}$. X-ray photoelectron spectroscopy (XPS) measurements were collected by using an ESCALAB MkII (VG Scientific) spectrometer, equipped with a standard $\mathrm{Al} \mathrm{K} \alpha$ excitation source and a 5-channeltron detection system for spectroscopic analysis. The pass energy was kept constant at $20 \mathrm{eV}$, and the analyzed area of the sample was about $5 \mathrm{~mm}$ in diameter. Spectroscopic data have been processed by the Avantage v. 5 software.

${ }^{13} \mathrm{C}$ Cross Polarization Magic Angle Spinning Nuclear Magnetic Resonance $\left({ }^{13} \mathrm{C}\{1 \mathrm{H}\}\right.$ CP-MAS NMR) spectra have been acquired at room temperature by a Bruker Avance II $400 \mathrm{MHz}(9.4 \mathrm{~T})$ spectrometer, operating at $100.63 \mathrm{MHz}$ for ${ }^{13} \mathrm{C}$. All experiments have been performed with a MAS spinning rate of $8 \mathrm{kHz}, 1024$ scans a contact time of $0.6 \mathrm{~ms}$, a delay time of $10 \mathrm{~s}$ and an excitation pulse on the ${ }^{1} \mathrm{H}$ nuclide of $4.5 \mathrm{~ms}$. A standard sample of adamantane has been used as an external reference and all samples were placed in a $4 \mathrm{~mm}$ zirconia rotors sealed with KEL-F caps. ${ }^{13} \mathrm{C}$ Direct Polarization (DP) solid state spectra with a MAS spinning rate of $8 \mathrm{kHz}, 1024$ scans, a delay time of $10 \mathrm{~s}$ and an excitation pulse on the ${ }^{13} \mathrm{C}$ nuclide of $4.5 \mathrm{~ms}$. A standard sample of adamantane has been used as an external reference and all samples were placed in a $4 \mathrm{~mm}$ zirconia rotors sealed with KELF caps.

Tapping mode amplitude modulation AFM measurements were performed by a Multimode V (Veeco Metrology) scanning probe microscope. The instrument was equipped with a conventional piezoscanner (maximum $\mathrm{xy}$ range $\approx 14 \mu \mathrm{m}$, and maximum $\mathrm{z}$ range $\approx 3.6 \mu \mathrm{m}$ ) and a four-segment photodetector for cantilever deflection monitoring. PointProbe®Plus Silicon-SPM-probes were used with Al backside reflex coating, resonance frequency $\approx 300 \mathrm{kHz}$ and tip apical diameter $\approx 10 \mathrm{~nm}$. All the scans were executed at room tem- 
perature and under $\mathrm{N}_{2}$ atmosphere. The inert gas ambient, obtained by continuous purging the sample chamber with $\mathrm{N}_{2}$ (flow rate $\approx 2 \mathrm{~L} \cdot \mathrm{min}^{-1}$ ), was necessary to reduce the probability of adhesion of the nanocomposite to the tip, by efficiently reducing the capillary forces acting between them.

SEM analysis was performed by scanning electron microscopy (ESEM FEI QUANTA 200). The samples were attached on an aluminum stub using an adhesive carbon tape and then sputter coated with gold (Sputtering Scancoat Six, Edwards) for $90 \mathrm{~s}$ under Ar atmosphere before imaging to avoid electrostatic discharge during the test. The surface composition was studied with a Phenom ProX, PhenomWorld equipped with EDX probe to detect the presence of $\mathrm{Si}$ and $\mathrm{N}$ in the insoluble fraction of nanocomposite after dissolution in formic acid at $80^{\circ} \mathrm{C}$ and subsequent centrifugation multiple-step (4 runs at $6000 \mathrm{rpm}$ for $20 \mathrm{~min}$ ).

Wide angle X-ray diffractometry (WAXD) analysis was conducted by using an Empyrean PANalytical II diffractometer with a $\mathrm{Cu} \mathrm{K} \alpha$ radiation source $(\lambda=1.5406 \AA)$.

Differential scanning calorimetry was carried out using a Shimadzu DSC 60 under $\mathrm{N}_{2}$ flow. The scanning rate was set to $10{ }^{\circ} \mathrm{C} /$ $\min$.

Thermogravimetric analysis (TGA) was carried out using a Mettler Toledo TGA/DSC instrument, all the experimental runs were performed under $\mathrm{N}_{2}$ flow $\left(100 \mathrm{ml} \cdot \mathrm{min}^{-1}\right)$ and two different temperature ramps: a slow ramp of $1{ }^{\circ} \mathrm{C} / \mathrm{min}$ from room temperature up to $800{ }^{\circ} \mathrm{C}$ and a faster ramp of $10^{\circ} \mathrm{C} / \mathrm{min}$ within the range $800-1400{ }^{\circ} \mathrm{C}$.

Thermo-dynamic-mechanical analysis was carried out by using a DMTA V Rheometrics Scientific equipment. The PA6 based nanocomposites (at least 3 replicates for each sample) were tested in the dual cantilever flexural mode. The specimen, $4 \mathrm{~mm}$ thick, $35 \mathrm{~mm}$ long and $8 \mathrm{~mm}$ wide, was clamped on the testing head and stressed with a small tensile pre-charge. Then it was inserted in an insulated thermostatic chamber under $\mathrm{N}_{2}$ flow. The test was carried out in temperature sweep mode in the range $30-180{ }^{\circ} \mathrm{C}$ at a heating rate of $5{ }^{\circ} \mathrm{C} /$ min. The maximum strain amplitude applied was $0.1 \%$ while the testing frequency was $1 \mathrm{~Hz}(6.28 \mathrm{rad} / \mathrm{s})$.

Tensile tests were performed on rectangular-shaped specimens cut off from compression-molded sheets $(10 \times 90 \times \sim 0.6 \mathrm{~mm})$. Mechanical testing was carried out by using an Instron 3365 universal tensile testing machine according to ASTM D882 on at least 10 replicates. The grip distance was set to $30 \mathrm{~mm}$ and the crosshead speed was $5 \mathrm{~mm} / \mathrm{min}$. The elastic modulus was calculated as the slope of each stress-strain curve extrapolated at zero-strain, toughness was calculated as the integrated area below stress-strain curves.

Impact tests (Izod mode) were carried out by a Ceast mod.6545 (Italy) universal apparatus on at least seven replicates of notched samples according to ASTM D256.

All the data were processed by calculating the average values and reporting the related error bars taking into account the data scattering.

\section{Results and discussion}

The characterization of GOS is briefly summarized in Fig. 1. The covalent attachment of silica to GO was demonstrated by FTIR/ATR, XPS and NMR spectroscopy. FTIR/ATR spectra collected for GO and GOS are shown in Fig. 1A. FTIR spectrum of GO displays a broad absorption band in the range $3750-3200 \mathrm{~cm}^{-1}$ assigned to $-\mathrm{OH}$ stretching due to phenols, carboxyls and water and two well recognizable bands, centered at around $1720 \mathrm{~cm}^{-1}$ and $1624 \mathrm{~cm}^{-1}$, with the former being assigned to carbonyls while the latter presumably originating by bending modes of water molecules integrated into graphenic sub-lattice $[23,24]$. At wavenumbers below $1250 \mathrm{~cm}^{-1}$ a bunch of overlapping signals was proposed to be due to $\mathrm{C}-\mathrm{O}-\mathrm{C}$ and $\mathrm{C}-\mathrm{OH}$ features, under the form of either epoxy, cyclic ethers and organic alcohols $[23,24]$. After the grafting of nanosilica, the bands assigned to $-\mathrm{OH}$ moieties (located at $1000 \mathrm{~cm}^{-1}$ and $3000-3600 \mathrm{~cm}^{-1}$ ) decrease, as well as those attributable to carboxyl and epoxy groups, whereas in the range $1200-900 \mathrm{~cm}^{-1}$ it is possible to detect a variegated absorption band with two main peaks. The one located at $1080 \mathrm{~cm}^{-1}$ can be attributable to the overlapping of $\mathrm{Si}-\mathrm{O}-\mathrm{Si}, \mathrm{C}-\mathrm{O}-\mathrm{C}$ and $\mathrm{Si}-\mathrm{O}-\mathrm{C}$ bonds (see blue-filled zone in the spectrum), while the peak centered at $1014 \mathrm{~cm}^{-1}$ can be reasonably assigned to silanols [22]. Furthermore, it is possible to recognize two bands attributable to -COOSi- bonding at $1488 \mathrm{~cm}^{-1}$ and $820 \mathrm{~cm}^{-1}$ (see orange-filled regions in GOS spectrum) [12,22,25].

Fig. 1B shows Si 2p XPS spectrum collected for GOS and silica. The results revealed that the combination of silica nanoparticles with GO took place via esterification and/or condensation mechanism, as indicated by the chemical shift registered in the Si $2 p$ signal and assigned to Si-O-C bond (binding energy $\mathrm{BE}=102.3 \mathrm{eV}$ ) [26]. The full elemental analysis of GO, nanosilica and GOS, provided in Table 1, allows estimating a degree of silica covalent immobilization equal to about $30 \%$.

Fig. 1C reports NMR results for GO and GOS. The GO spectrum collected in cross polarization $\mathrm{C}-\mathrm{H}$ evidenced the presence of carboxylic functionalities at about $160 \mathrm{ppm}$ and $\mathrm{C}-\mathrm{O}$ (ascribed to alcohols and epoxy) at about $70 \mathrm{ppm}[3,27] . \mathrm{sp}^{2}$ carbons related to the graphenic matrix are located in the range $110-150 \mathrm{ppm}$ in the cross polarization spectrum but no typical peak of graphitic carbon was detected, likely because of the high distance between $\mathrm{sp}^{2}$ carbons and the protons, thus suggesting a high degree of oxygen-functionalization and the presence of a variegated aromatic environment, constituted by ketones, lactols and small graphenic domains [22]. On the other hand, the presence of silica nanoparticles covalently linked to the GO lamellae, did not allow collecting for GOS a C-MAS spectrum via cross polarization, hence the spectrum reported was obtained in direct polarization on ${ }^{13} \mathrm{C}$. GOS spectrum shows the presence of a very intense

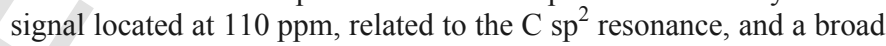
signal located at 59 and $71 \mathrm{ppm}$, mainly attributed to C-O-C, thus confirming that most of alcoholic, epoxy and carboxylic moieties reacted with silica.

TGA results, plotted in Fig. 1D, put into evidence the enormously increased thermal stability of GOS nanohybrids with respect to pristine GO. This feature plays a crucial role in the perspective of adopting a melt processing route. At $\mathrm{T}=240{ }^{\circ} \mathrm{C}$, i.e. the processing temperature chosen for the preparation of PA6-based nanocomposites, the residual mass percentage is approximately $87 \%$ for GOS while it is only $50 \%$ for GO [28].

The morphology of GOS, GO and nanosilica was carefully investigated. In Fig. 2A and D there are reported the AFM micrographs of GO (Fig. 2A), nanosilica (Fig. 2 B) and GOS (Fig. 2 C-D). A total exfoliation of GO into monolayered lamellae can be achieved after a prolonged sonication treatment (up to $36 \mathrm{~h}$ ), which is usually found to reduce $\mathrm{GO}$ size while promoting the formation of defects and holes, as already reported in the scientific literature [29].

Nanosilica aggregates constituted by spherical nanoparticles can be easily recognized in Fig. 2B. The surface investigation of a GOS flake dispersed after mild sonication gives no significant information, even if the silica layers covering the graphenic lamellae are well-recognizable. In order to assess qualitatively the strength of GO-silica bonding, a prolonged ultrasonic treatment was carried out even in this case. The results, provided in Fig. 2D, show a discrete distribution of silica nanoparticles that remain attached onto the basal planes of GO, 


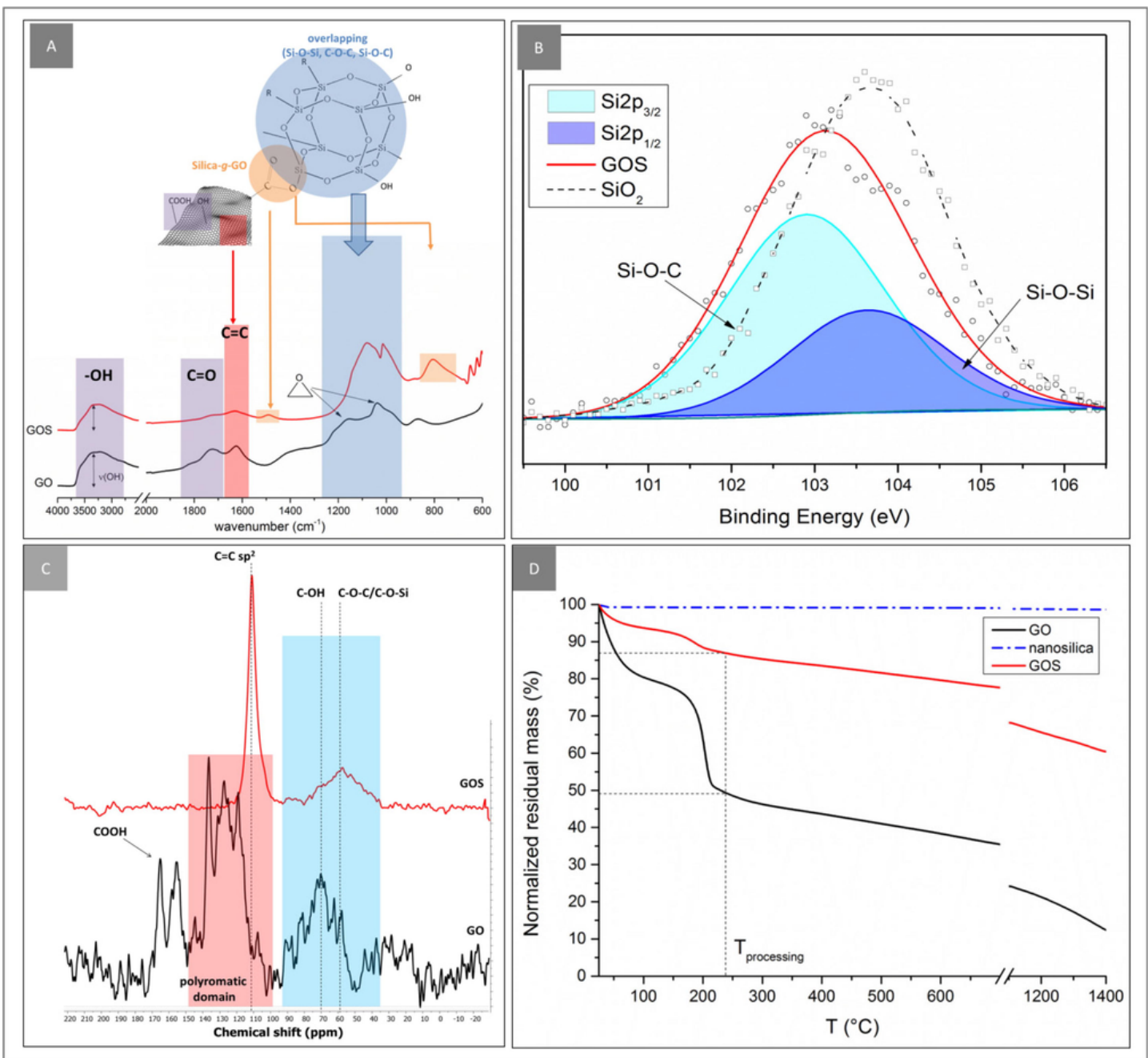

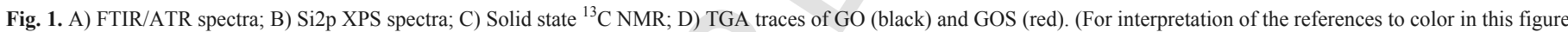
legend, the reader is referred to the web version of this article.)

Table 1

Elemental analysis from XPS.

\begin{tabular}{|c|c|c|c|c|c|c|c|c|c|c|c|}
\hline & & \multicolumn{5}{|l|}{$\mathrm{C}$} & \multicolumn{3}{|l|}{$\mathbf{O}$} & \multicolumn{2}{|l|}{$\mathbf{S i}$} \\
\hline \multirow{4}{*}{ GO } & At.\% & \multicolumn{5}{|l|}{51.4} & \multicolumn{3}{|l|}{48.6} & \multicolumn{2}{|l|}{-} \\
\hline & & \multicolumn{3}{|l|}{19.5} & \multicolumn{2}{|c|}{5.6} & \multicolumn{3}{|l|}{43.6} & - & - \\
\hline & $\mathrm{BE}(\mathrm{eV})$ & \multicolumn{2}{|l|}{284.5} & 287.0 & \multicolumn{2}{|c|}{289.3} & 532.3 & \multicolumn{2}{|l|}{534.8} & - & - \\
\hline & & \multicolumn{2}{|l|}{ C-C } & $\mathrm{C}-\mathrm{O}$ & \multicolumn{2}{|c|}{-COO- } & $\begin{array}{l}\mathrm{C}-\mathrm{O}, \\
\mathrm{H}_{2} \mathrm{O}\end{array}$ & \multicolumn{2}{|l|}{ Oxide } & - & - \\
\hline & At.\% & \multicolumn{5}{|l|}{4.4} & \multicolumn{3}{|l|}{59.0} & \multicolumn{2}{|l|}{36.6} \\
\hline \multirow{4}{*}{ Silica } & & \multicolumn{3}{|l|}{2.5} & \multicolumn{2}{|l|}{-} & 52.0 & \multicolumn{2}{|l|}{7.0} & 13.9 & 3.5 \\
\hline & $\mathrm{BE}(\mathrm{eV})$ & \multicolumn{2}{|l|}{285.0} & 286.7 & \multicolumn{2}{|c|}{-} & \multirow{2}{*}{$\begin{array}{l}533.5 \\
\mathrm{SiO}_{2}\end{array}$} & \multicolumn{2}{|l|}{534.9} & 104.4 & 105.8 \\
\hline & & C-C & & $\mathrm{C}-\mathrm{O}$ & & bO- & & Dymethyle & rbonate & $\mathrm{Si}^{4+}$ & $\mathrm{SiMe}_{4}$ \\
\hline & & \multicolumn{5}{|l|}{ C } & & $\mathbf{S i}$ \\
\hline & At.\% & 51.8 & & & & & & & & & 10.2 \\
\hline & & 25.7 & 19.2 & 5.5 & & 1.5 & 11.6 & & 22.5 & 3.8 & 10.2 \\
\hline GOS & $\mathrm{BE}(\mathrm{eV})$ & 285.0 & 287.1 & 28 & & 290.5 & 534.7 & & 533.2 & 531.4 & 102.9 \\
\hline & & C-C & $\mathrm{C}-\mathrm{O}$ & & & $-\mathrm{CO}_{3}$ & Dyme & ylcarbonate & $\begin{array}{l}\mathrm{C}-\mathrm{O} ; \\
\mathrm{H}_{2} \mathrm{O}\end{array}$ & $\mathrm{Si}-\mathrm{O}$ & Si-O-C \\
\hline
\end{tabular}




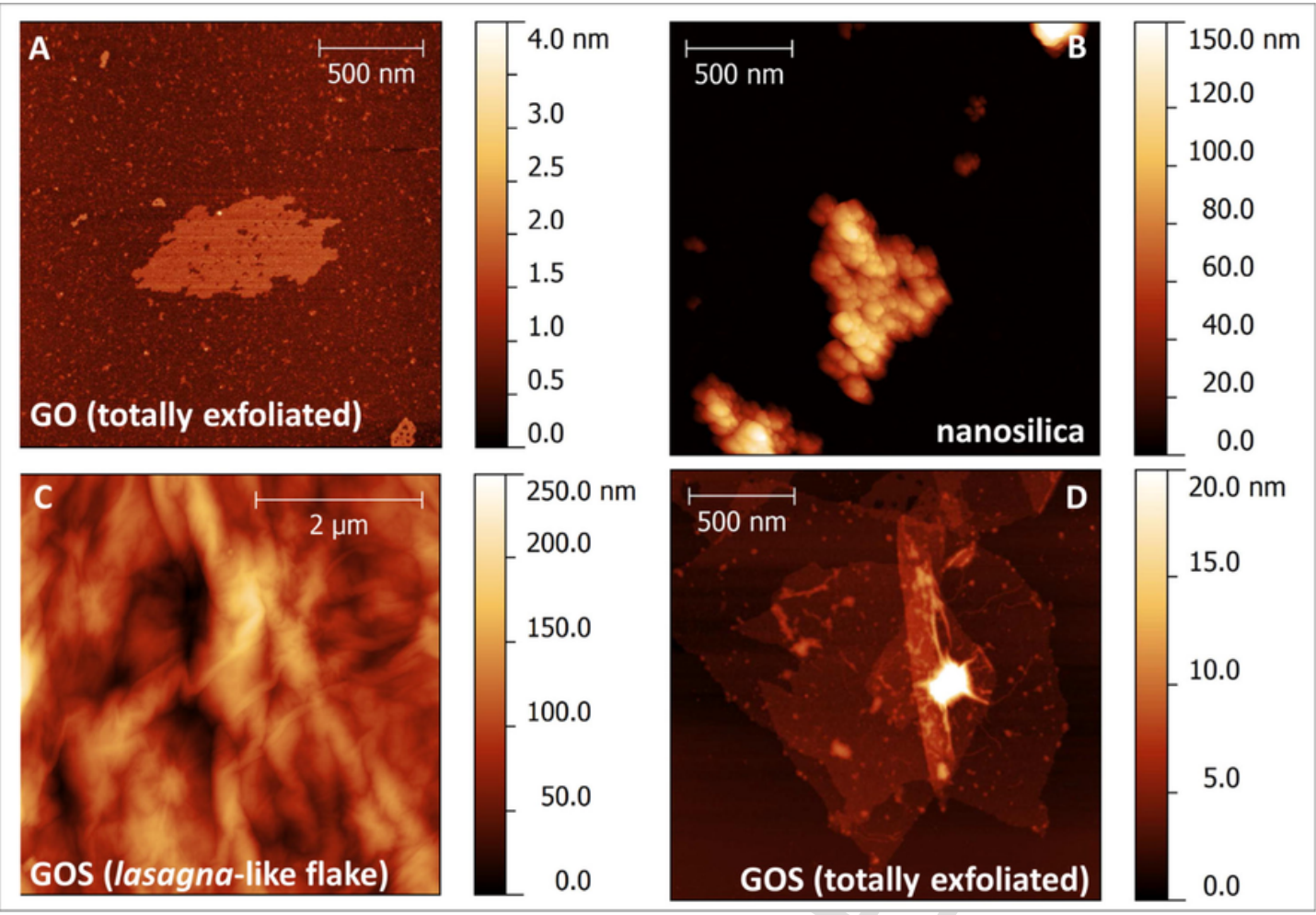

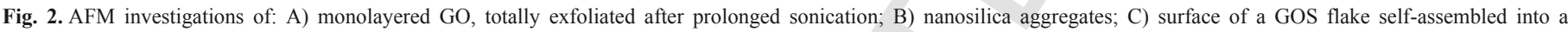
lasagna-like structure and D) totally exfoliated after prolonged sonication.

thus suggesting that graphenic silicates are quite stable. Interestingly, the AFM features of GOS lamellae herein synthesized appear to be very similar to those of GO-silica nanohybrids achieved in different ways [20].

SEM investigation of GOS was found to be particularly suitable to describe the lasagna-like structure of these nanohybrids. Fig. 3A and D provides different magnifications of a GOS flake cross-section, whereas it is possible to observe the macroscopic structure, made by GO lamellae intercalated by silica layers (panel A), the adhesion of silica nanoparticles onto graphenic planes (panel B) and the exfoliation of GO layers, surrounded by silica (panel C). The exfoliation of $\mathrm{GO}$ was further confirmed by XRD analysis, reported in Fig. 3D. In fact, the $\left(\begin{array}{lll}0 & 0 & 1\end{array}\right)$ diffraction peak of GO totally disappears after conjugation with silica, while the amorphous silica peak remains, although slightly shifted and broadened, suggesting a strong GO-silica interaction.

Fig. 4 provides a synoptic pictorial representation of the processing-structure relationship for the systems studied in the frame of this work, together with AFM images of GO, silica, GOS and SEM micrographs of polymer-based nanocomposites containing GO (Fig. 4-A), GO plus silica (Fig. 4-B) and GOS (Fig. 4-C).

Directly melt mixing PA6 and GO (Fig. 4-A) causes the insurgence of pleating and crumpling phenomena, as previously debated, leading to the formation of stacked clusters (tens of $\mu \mathrm{m}$ in size) that reflects the expected problems of poor dispersion. Nevertheless, the variegated oxygen moieties bearing onto basal planes and at the edges of GO determine an excellent interfacial adhesion with PA6. GO, indeed, is able to chemically react with $-\mathrm{NH}_{2}$ end groups of PA6 by exploiting its carboxyl and epoxy moieties and/or to strongly interact with the polymer by H-bridge linkage [4]. On the other hand, when GO is fed to the mixer in the solid state, the fast kinetics of self-aggregation lead to a bunch of inter- and intra-lamellar reactions involving epoxy, alcohols and carboxyl moieties via condensation mechanisms, which determine the crumpling and/or the folding of lamellae into micro-sized aggregates. As a matter of consequence, any benefit related to the strong filler-matrix affinity is substantially lost because of the lack of dispersion and PA6-GO can be considered a micro-composite, rather than a nanocomposite.

Similarly to GO, even silica nanoparticles are known to show high affinity to polyamides, since amino groups of PA6 enter into strong interactions with the surface of silica via Lewis acid-base complexing, H-bonds and Brønsted acid-base reactions [30,31]. Nevertheless, when GO, silica and PA6 are fed together in the mixer (Fig. 4-B), the aggregation of GO lamellae is still relevant. Moreover, in this case silica-filled GO sacks were detected, with big aggregates of crumpled GO lamellae surrounding silica clusters and polymer (see further the EDX mapping conducted onto extracted clusters). This phenomenon was somehow similar to that discovered by Chen et al. in the case of GO co-suspended with other nanoparticles in dilute aqueous phase, nebulized and heated [32]. Interestingly, when GOS is used as a filler (Fig. 4-C), the morphology drastically changes. GOS are built by exfoliated GO lamellae with a degree of covalent silica-functionalization equal to $30 \%$ (atomic percentage). The remaining silica nanoparticles were found to intercalate between lamellae and to stratify into silica-layers due to the condensation reactions involving adjacent silanol groups to yield siloxane. Under the shear stresses present in a batch mixer, silica nanoparticles not covalently bonded tend to disperse within the matrix, as well as the silica-decorated GO lamellae. The main goal of this pathway lies in the possibility to reduce or even stop the self-aggregation of GO sheets, thus maximizing the dispersion. Herein, we achieved a three-component system with a polymeric phase and two different fillers, i.e. lamellar GOS and spherical silica nanoparticles. 


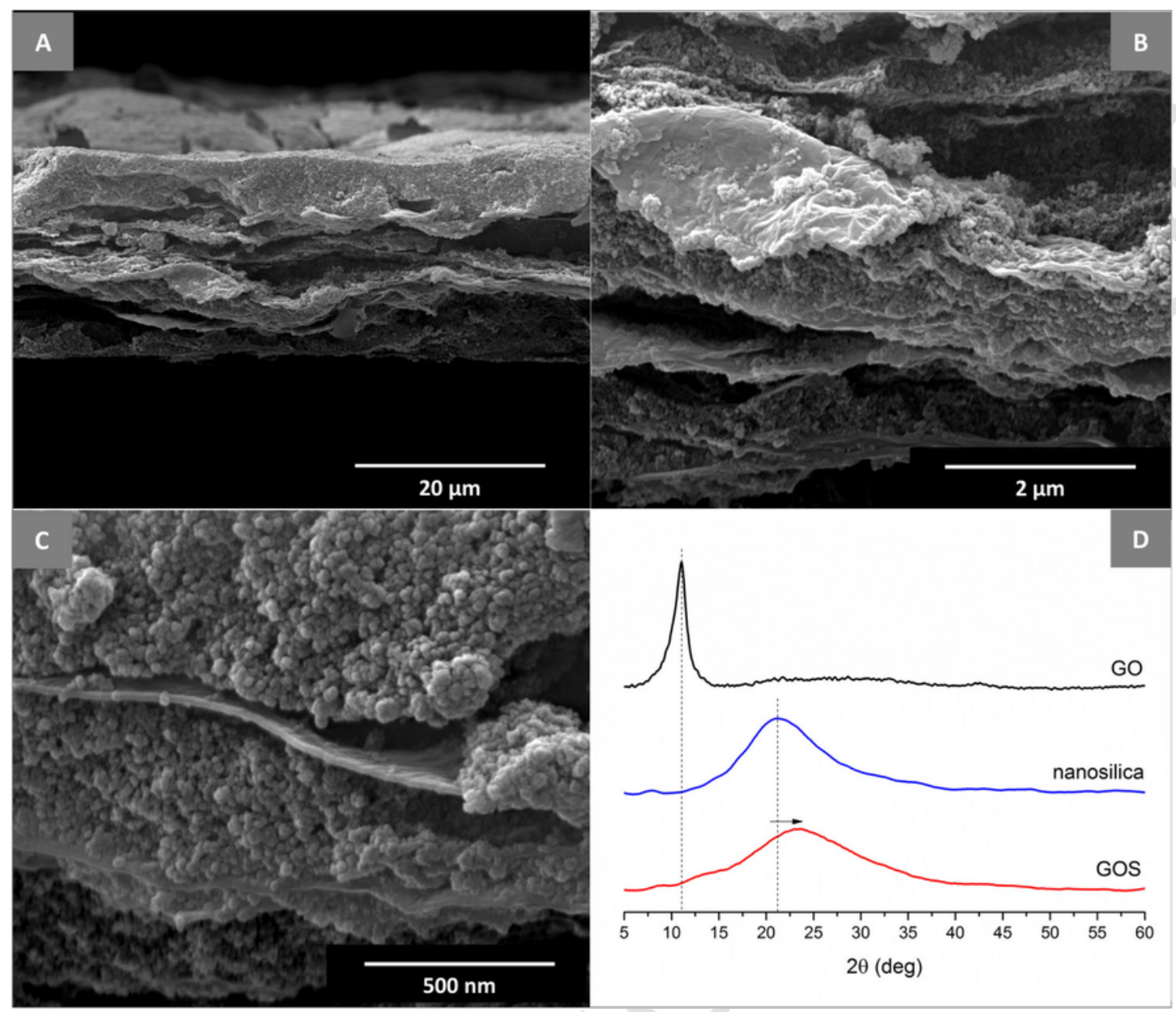

Fig. 3. SEM micrographs at different magnifications of GOS flakes assembled into a lasagna-like (A-C); XRD patterns of GO, nanosilica and GOS nanohybrids (D).

DMA tests were performed to get information about either the thermo-mechanical properties of the materials and the interphase [33]. Fig. 5A shows the storage modulus (E') as a function of temperature for each sample prepared in the frame of this work. E' increases in the order: $\mathrm{PA} 6<\mathrm{PA} 6+\mathrm{GO}<\mathrm{PA} 6+\mathrm{GO}+\mathrm{S}<\mathrm{PA} 6+\mathrm{GOS}$. Moreover, as regards the systems containing GOS, a sharp effect of filler concentration can be observed, since adding $0.5 \%$ of GOS leads to a storage modulus in the glassy region close to $3 \mathrm{GPa}$ (about a threefold increase with respect to neat PA6), whereas loading $0.25 \%$ of GOS determines a storage modulus of about $2 \mathrm{GPa}$ (twofold increase if compared to the pure matrix). Furthermore, heat distortion temperature (HDT), calculated according to Takemori's method [34] is provided within the same plot. HDT was found to increase from $51{ }^{\circ} \mathrm{C}$ of the pure polymer to $94{ }^{\circ} \mathrm{C}$ of the PA6 containing $0.5 \%$ of GOS, thus improving its poor overall thermomechanical resistance.

In order to give further insight into the effect of interphase, the damping factor (i.e. tan delta) from DMA analysis was carefully examined by taking into account three key-parameters of loss factor plot, such as intensity, FWHM and peak temperature, particularly useful to get information about interfacial adhesion, volume of interphase and interaction, respectively [35].

Fig. 5B provides the loss factor plots of PA6+GOS nanohybrid composites, together with those of neat polymer and of systems containing $\mathrm{GO}$ and $\mathrm{GO}+\mathrm{S}$, for sake of comparison. PA6 shows a well pronounced peak centered at $68^{\circ} \mathrm{C}$, ascribed to the glass transition of the amorphous aliquot of macromolecules [5]. The addition of $0.5 \%$ GO (wt/wt) determines a sharp peak-shift towards higher temperature and a depletion in the height of $\tan \delta$, symptomatic of a stronger interfacial adhesion with the polymer $[33,35,36]$. However, the bad dispersion leaves substantially unaltered the FWHM of loss factor band, thus suggesting that the volume of confined macromolecules is low. When GO and silica are mixed together with the host polymer, analogue considerations can be made, even if intensity of $\tan \delta$ is found to slightly decrease. On the contrary, the system constituted by PA6 and GOS, shows several differences with respect to those containing GO or $\mathrm{GO}+\mathrm{S}$.

In fact, the height of $\tan \delta$ was found to drastically decrease, thus suggesting that the macromolecules are strongly bound with hybrid nanoparticles. Furthermore, the remarkable broadening of FWHM observed even at a loading level as low as $0.25 \%$ - seems to prove that the volume of macromolecules confined, i.e. the volume of interphase, increases with respect to the other systems.

These features can be likely ascribed to a high degree of exfoliation of GO, owing to the interplay between the shear forces due to melt mixing and the ability of silica layers to prevent the re-aggregation of lamellae. Weakly bound layers of silica nanoparticles are supposed to behave as solid-state exfoliating agents, while those covalently attached to lamellae could have hindered crumpling phenomena [37-39]. All of these features contribute to provide a remarkable reinforcing effect, probably enabling the formation of a robust, ex- 


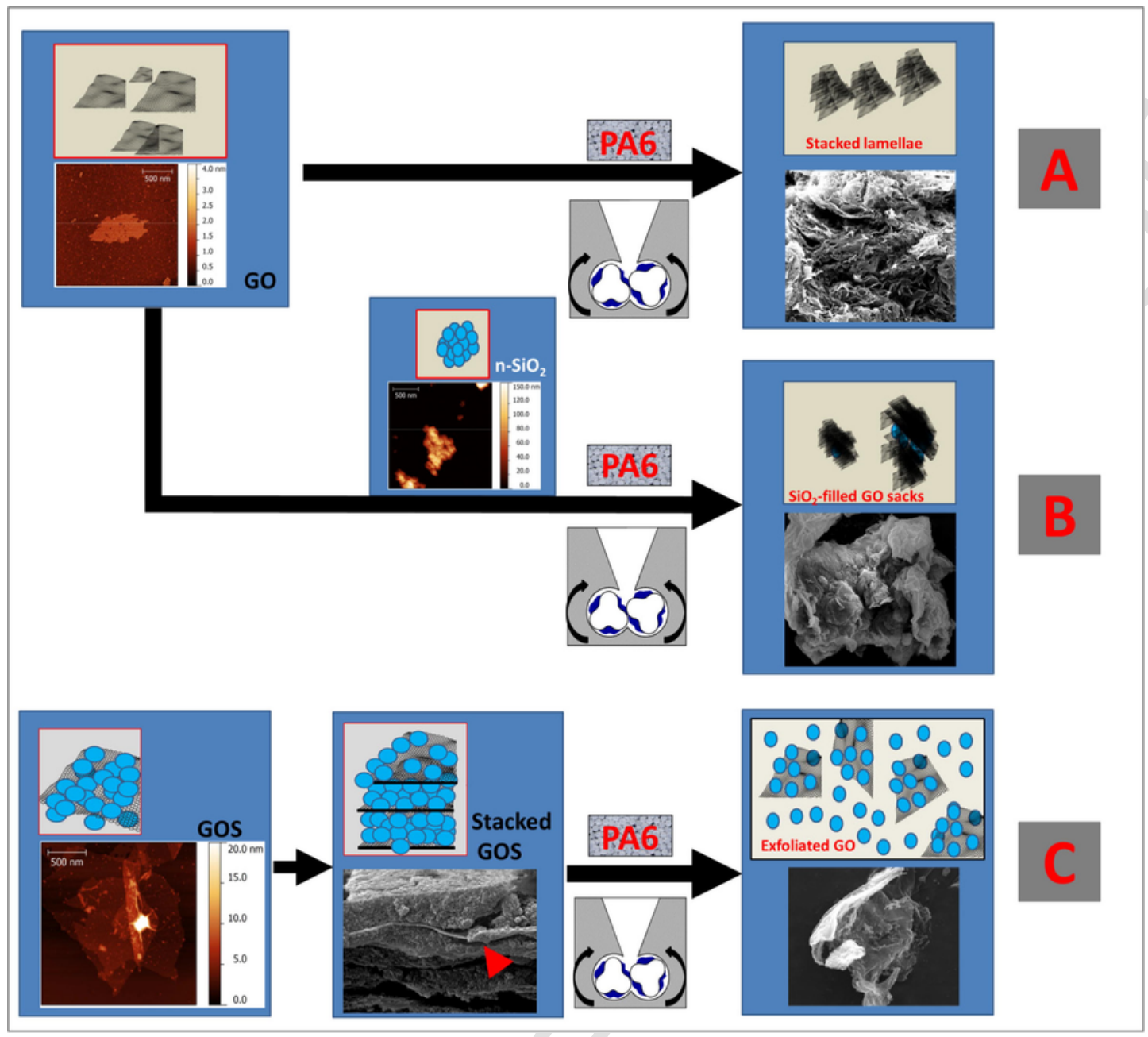

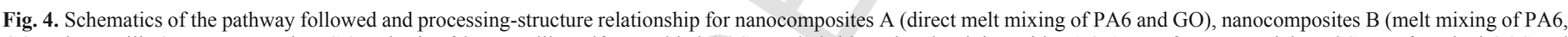

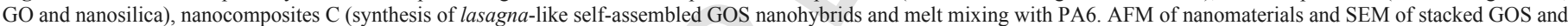
polymer-based nanocomposites are provided together with the pictorial representation.

tended and variegated interphase region, as suggested by the different shape of tan delta plots in PA6-GOS system, which displays a well recognizable double peak. This latter feature was even assessed by examining multi-peak fitted loss factor plots of the systems containing $0.25 \%$ and $0.5 \%$ GOS, respectively, shown in Fig. 5C and D. Both curves exhibit at least two main contributions: (i) one peak located at about $70-80{ }^{\circ} \mathrm{C}$, basically similar to those of PA6, PA6-GO and PA6-GO+S, although slightly up-shifted, (ii) a broad contribution centered at about $120^{\circ} \mathrm{C}$. These outcomes seem to suggest that in PA6-GOS system, most of macromolecules undergoes well-distinct transitions at such higher temperatures than pure polymer, likely owing to the formation of a multiple interphase [40].

The cross-section of cryofractured samples is shown in Fig. 6. The choice of some key-parameters, such as the use of silica as pre-dispersant and the type of feeding, clearly affected the morphology of the system. PA6+GO displays re-stacked crumpled GO, assembled into a honeycomb-like structure, as a result of the aforementioned thermally-driven intra- and interlamellar reactions. Similarly, the system PA6+GO+S exhibits micrometric-sized aggregates but slightly different in feature. However, a good interfacial adhesion between polymer and clusters seems to be suggested by the presence of polyamide fibrils in this latter case. On the contrary, when GOS is used as a nanofiller, no aggregates were detected and the surface was found to be more uniform, in fully agreement with the increase of tan delta FWHM. Moreover, Fig. 7 reports a detailed inspection of emerging nanohybrid structures constituted by GOS lamellae totally wetted by polymer. This feature was found at both loading levels tested in the frame of this work and it reveals a strong interfacial adhesion between polymer and GOS, in agreement with the reduced loss factor intensity registered for PA6-GOS system during DMA experiments.

In order to perform a closer investigation of interphase, the samples were dissolved in formic acid at $80^{\circ} \mathrm{C}$ and repeatedly centrifuged to extract free polymer chains, while the insoluble fraction of each sample was collected and analyzed. Fig. 8 reports the micrographs of interphase extracted from $\mathrm{PA} 6+\mathrm{GO}+\mathrm{S}$ and $\mathrm{PA} 6+0.5 \% \mathrm{GOS}$, together with EDX mapping of polymer and silica, easily recognizable by monitoring the signals of $\mathrm{N}$ and $\mathrm{Si}$ elements, respectively. The GO-sacks extracted from $\mathrm{PA} 6+\mathrm{GO}+\mathrm{S}$ (top left micrograph) are constituted by crumpled lamellae of graphene oxide. They were found to contain both PA6 likely reacted with graphene oxide and silica aggregates (see bottom left picture), thus confirming that the fillers show a good affinity towards the matrix, despite their poor dispersion. On the contrary, PA $6+0.5 \%$ GOS shows a thick layer of polymer surrounding well-embedded nanoparticles. In this latter case, 

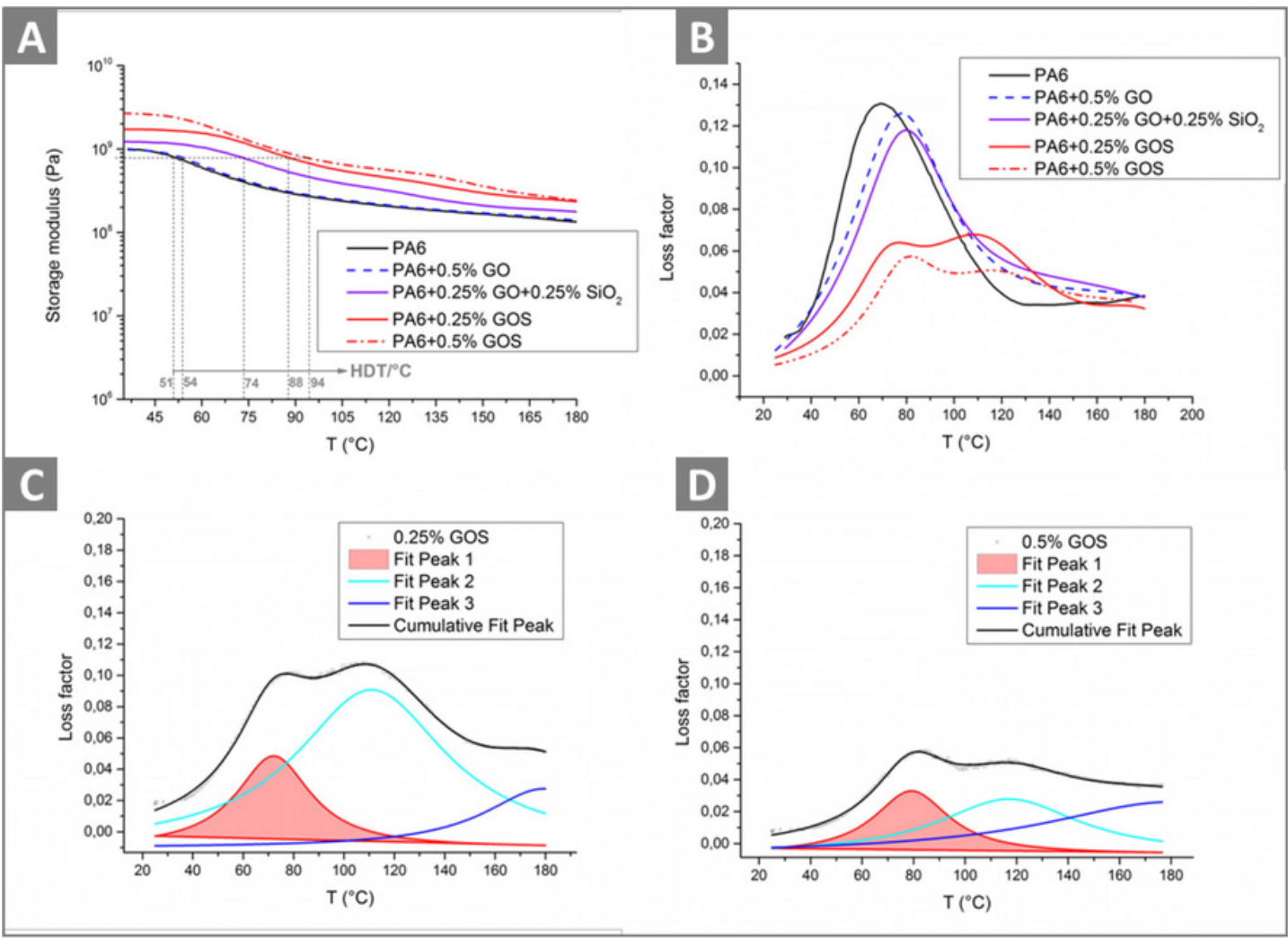

Fig. 5. DMA results: Storage modulus plotted as a function of temperature (together with HDT) for each sample investigated (A); Loss factor plotted as a function of temperature for all the systems investigated (B); Multi-peak fitted loss factor plots of systems containing $0.25 \%$ (C) and $0.5 \%$ (D) of GOS.

the interphase region is clearly much more extended and the presence of polymer, as well as of the silica nanoparticles, is uniform (see bottom right picture).

The structure of nanocomposites was also studied by WAXD investigations. The outcomes, reported in Fig. 9, put into evidence that both $\alpha_{1}$ and triclinic $\gamma$-form slightly increase when GOS is added, especially at the highest loading investigated (i.e. $0.5 \%$ ). As commonly accepted, $\alpha_{1}$ and $\gamma$-phase crystallites may provide higher stiffness respectively below and beyond $\mathrm{T}_{\mathrm{g}}$, with this latter feature imparting higher HDT [41]. DSC traces of PA6 and of the nanocomposites collected within cooling and heating scans are reported in Fig. 10A and B, respectively. The results put into evidence that no appreciable differences are detectable in all the systems investigated in terms of $\mathrm{T}_{\mathrm{c}}$ and $\Delta \mathrm{H}_{\mathrm{c}}$. As regards the second heating scan, PA6 and $\mathrm{P} 6+\mathrm{GO}$ exhibit a double peak centered at $\mathrm{T}_{\mathrm{ml}}=212.5^{\circ} \mathrm{C}$ and $\mathrm{T}_{\mathrm{m} 2}=220^{\circ} \mathrm{C}$, ascribed to melting points of $\alpha_{1}$ and $\alpha_{2}$ crystallites, respectively. The sample $\mathrm{PA} 6+\mathrm{GO}+\mathrm{S}$ displays a similar behavior, although $\mathrm{T}_{\mathrm{m} 1}$ peak was found to shift to $215^{\circ} \mathrm{C}$. The traces registered for system PA6+GOS show a slightly different behavior, since no peak was detected at $\mathrm{T}=212-215^{\circ} \mathrm{C}$, whereas in the temperature range $219-225^{\circ} \mathrm{C}$ the curves show a variegated region, presumably associated to the overlapping of melting enthalpies related to $\gamma$ and $\alpha_{2}$ crystallites. Furthermore, the offset temperature $\left(\mathrm{T}_{\text {offset }}\right)$ was equal to 225-227 ${ }^{\circ} \mathrm{C}$, slightly shifted with respect to those of pure PA6 and other nanocomposites $\left(\mathrm{T}_{\text {offset }}=223^{\circ} \mathrm{C}\right)$. All of these outcomes suggest that a moderate nucleating effect is imparted by GOS, as well as higher quality of crystallites, thus leading to higher melting temperatures. The degree of crystallinity $\left(\chi_{\mathrm{c}}\right)$, being equal to $14 \%$ for neat PA6, slightly increased to $18-19 \%$ when GO and GO+S were added, whereas was found to increase about twofold $\left(\chi_{\mathrm{c}}=23-24 \%\right)$ in the case of PA6+GOS samples.

Therefore, the increased crystallinity (and polymorphism) of PA6 in presence of GOS could have actively contributed to the enhancement of thermo-mechanical properties exhibited by PA6-GOS nanocomposites. Nonetheless, due to the small changes observed in crystallinity either by WAXD or DSC, the stiffening registered below and over $\mathrm{T}_{\mathrm{g}}$ seem to be mainly governed by the interphase region rather than crystallinity.

The representative stress-strain curves of all the samples investigated are provided in Fig. 11, whereas the salient mechanical results carried out from tensile and IZOD tests, such as elastic modulus, tensile strength, elongation at break, toughness and impact strength are listed in Table 2. Basically, these results confirm those already commented. Indeed, both PA6+GO and PA6 $+\mathrm{GO}+\mathrm{S}$ samples display moderately higher stiffness than pure polymer, due to re-aggregative phenomena involving GO lamellae, despite a conspicuous strengthening effect, likely promoted by a strong interfacial adhesion. The clusters resulting from folding and crumpling phenomena dramatically affect EB, toughness and impact strength [42].

On the other hand, when GOS is used as a hybrid filler, both stiffness and tensile strength are found to dramatically increase, while elongation at break substantially remains unaltered and close to that of pure matrix.

Based on these observations, it can be concluded that the interfacial adhesion is strong in all the nanocomposites investigated but, conversely, the poor dispersion limits the benefits associated to the presence of GO and silica. Differently, systems containing GOS take advantage from the better dispersion, thus exhibiting higher mechanical performance. Indeed, previous reports indicate that at the ex- 

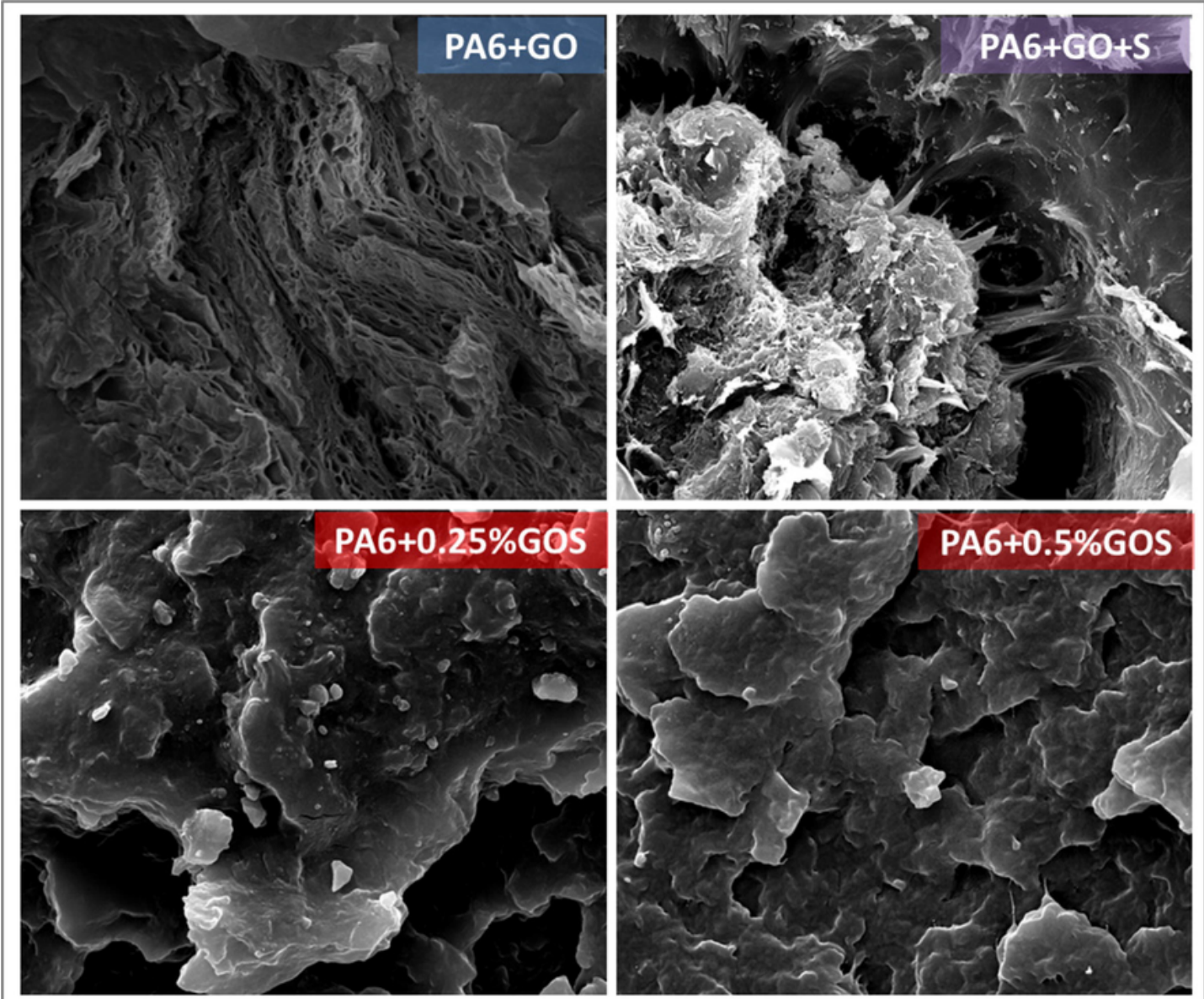

\section{$20 \mu \mathrm{m}$}

Fig. 6. Cryofractured cross-section of the nanocomposites investigated (scale bar $=20 \mu \mathrm{m}$ )

tremely low filler loadings used in the frame of this work, the two nanoparticles separately used show negligible effects on mechanical and thermomechanical properties of PA6-based nanocomposites, due to lack of dispersion [5,30,31]. Interestingly, the nanodomains originated by GOS dispersion within host polymer either constrain the polymer chains and enhance the toughening efficiency of the polymer due to the singular microstructure which is ensured by a variegated interphase region where GO lamellae and silica nanospheres synergistically interact with the matrix by an interlocking mechanism $[34,35]$. Therefore, the processing-structure-properties relationship, unambiguously assessed in this work, provides guidance to future advances in fabricating a new generation of graphene-based ternary nanohybrids in the melt.

In fact, according to the existing literature, PA6-GO nanocomposites have been prepared by adopting protocols requiring a time consumption ranging from 8 to almost $100 \mathrm{~h}$ (taking into account synthetic plus drying/pre-treatment conditions) and the use of chemicals, as solvents and/or reactants to chemically functionalize GO and/or PA6 $[3,43,44]$. Despite the long time protocols and the environmental risks, the extent of mechanical improvement ranges from $10 \%$ to $300 \%$. Only few papers report on the simultaneous stiffening, strengthening and toughening of PA6 with the incorporation of a filler content as low as $0.25 \%$ or $0.5 \%$ (which in turns corresponds to a GO content equal to $0.125 \%$ and $0.25 \%$, respectively).

Even when compared to the techniques employed to prepare other types of PA6-GO-ceramics nanocomposites [14,15], the route herein presented requires less time, lower temperatures and remarkably lower amounts of water.

\section{Conclusion}

Ternary nanohybrids based on PA6, graphene oxide and silica were successfully prepared by melt mixing the polymer with a water-prepared graphene oxide-silica nanohybrid filler and compared with the nanocomposites achieved by loading GO and GO plus silica $(\mathrm{GO}+\mathrm{S})$ in the solid state.

This novel strategy can be considered green for several reasons. First, this technique drastically reduces the time and the difficulty related to solvent (amounts used, removal, recycling, disposal, etc.), avoids water consumption, often necessary in the case of wet phase inversion or for other green approaches involving reactions in aqueous environment, since in this case $150 \mathrm{~mL}$ of water under mild operative conditions $\left(\mathrm{T}=120^{\circ} \mathrm{C}\right.$ for about $30 \mathrm{~min}$ at atmospheric pressure) are enough to prepare the GOS amount necessary for the realization of almost $100 \mathrm{~g}$ of PA6-GOS nanocomposites. These latter 


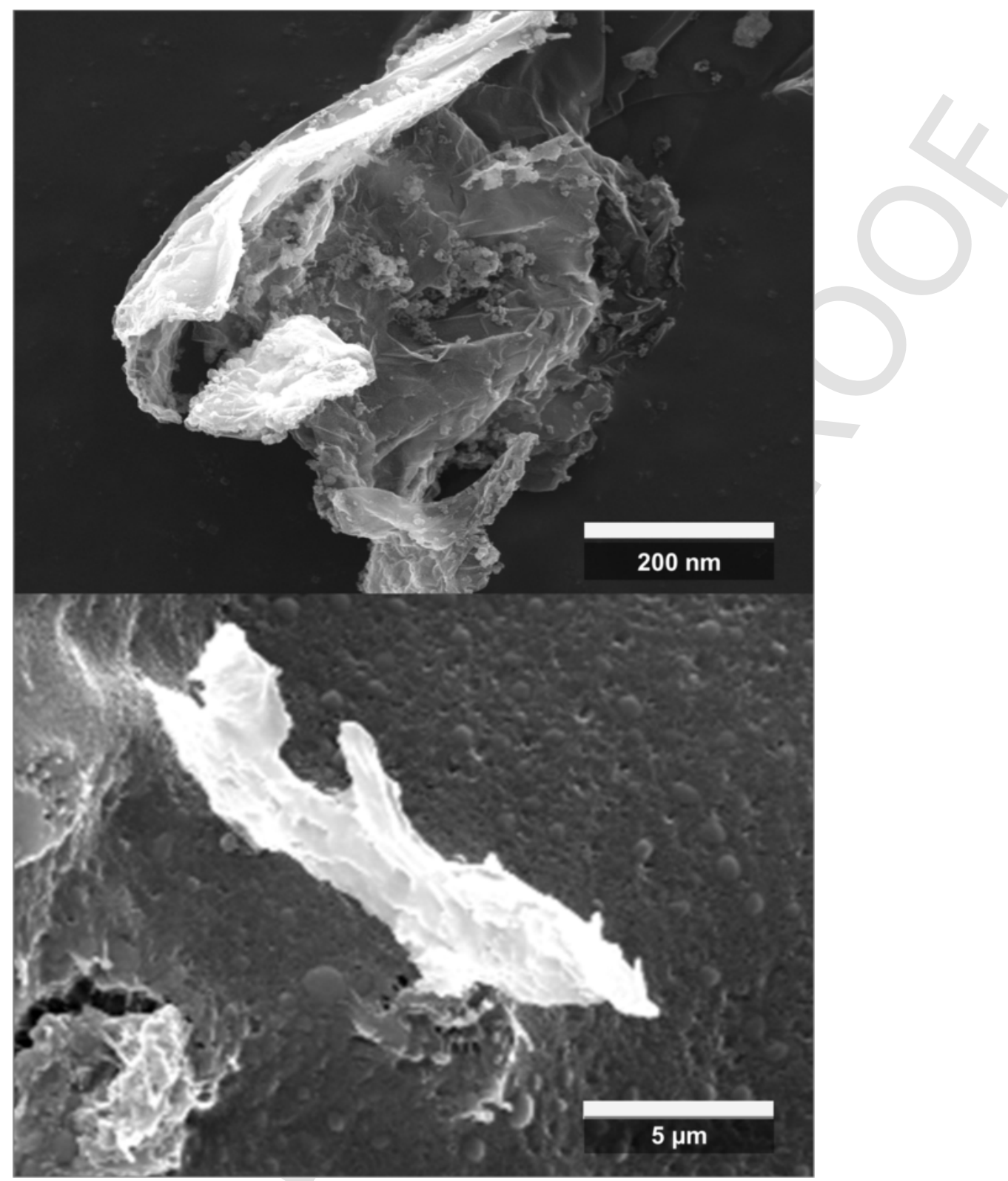

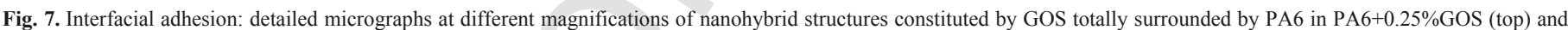
PA6+0.5\%GOS (bottom)

ones exhibit strong mechanical and thermomechanical performance. In summary, PA6-GOS nanohybrids have a HDT $40^{\circ} \mathrm{C}$ higher than pure polymer, thus overcoming the well-known poor thermomechanical stability of polyamide 6 . Furthermore, adding GOS provides the simultaneous stiffening (up to $+180 \%$ ), strengthening (up to $+210 \%$ ) and toughening (up to $+210 \%$ ) of the polymer, by substantially pre- serving its ductility. The excellent enhancement of mechanical and especially thermomechanical performance observed with adding only $0.5 \mathrm{wt} \%$ of GOS opens new scenarios for this class of nanostructured integrated materials to be used in the field of high performance fibers and/or engineering materials, such as devices for high-temperature applications or structural materials for aerospace engineering. 

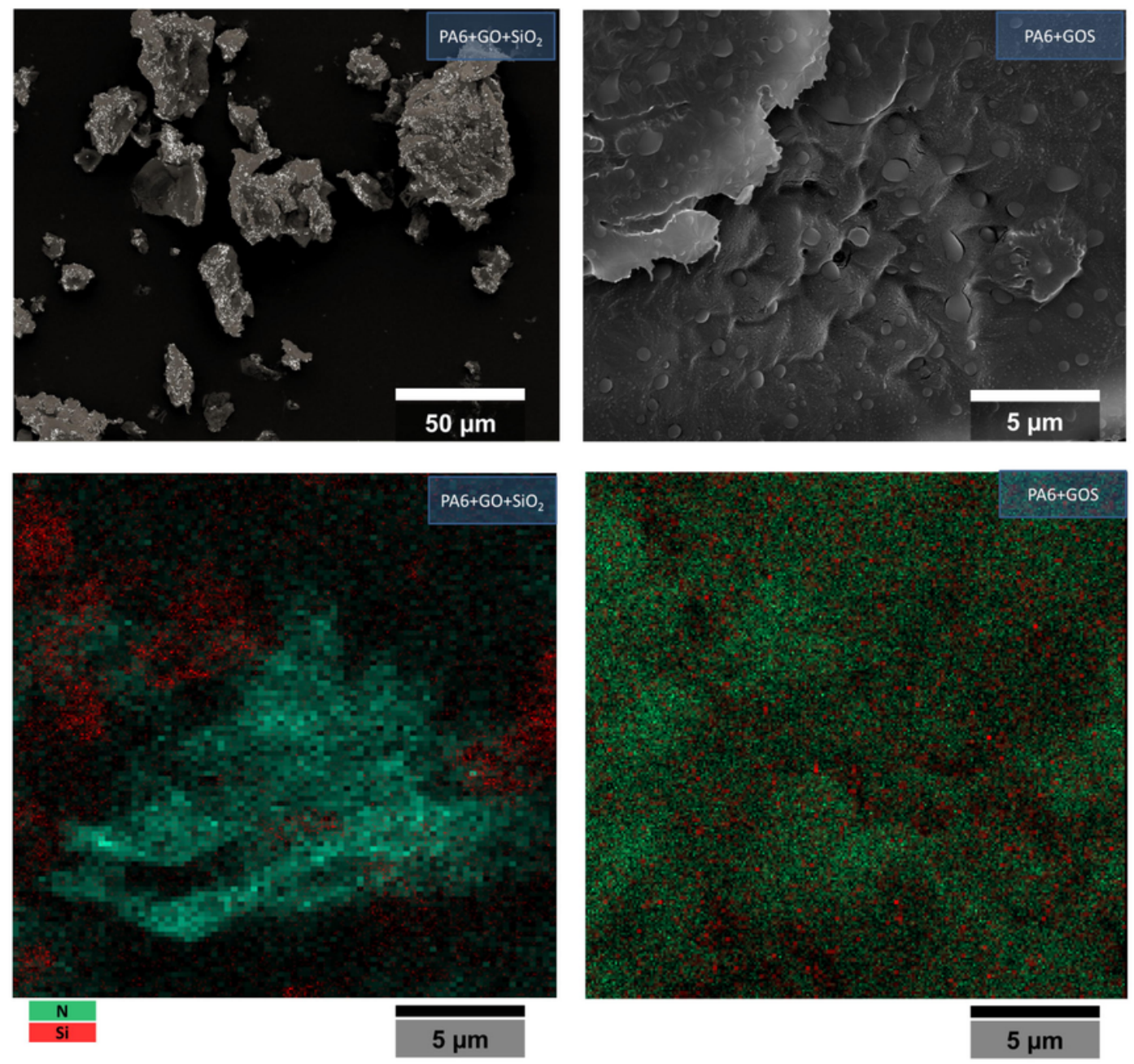

Fig. 8. Interphase study: Silica and Polymer distribution within the insoluble interphase extracted by centrifugation in formic acid for PA6+GO+S and PA6+0.5\%GOS. Silica and polymer were monitored by SEM and EDX mapping of Si and N elements. 


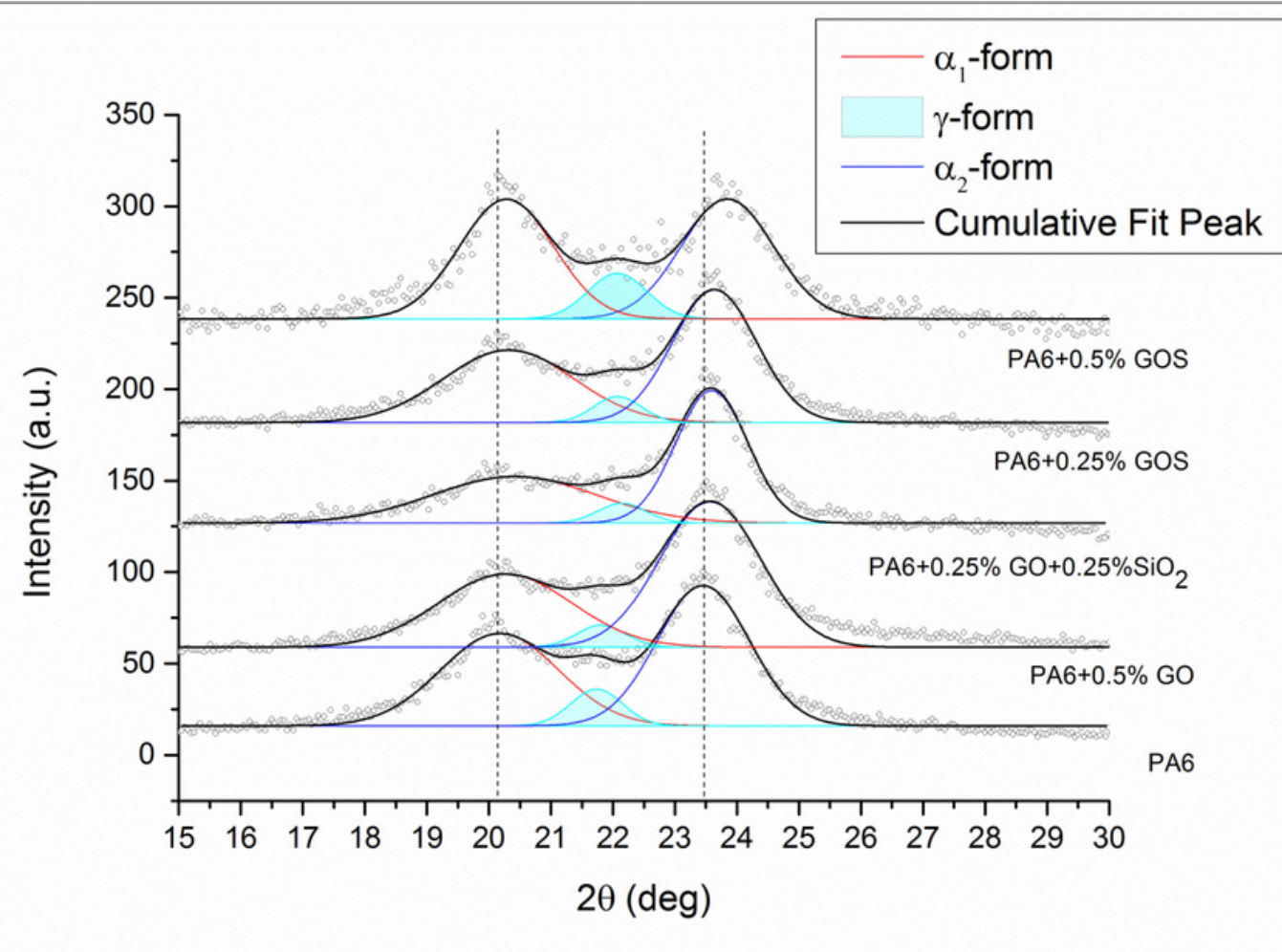

Fig. 9. WAXD traces of nanocomposites and multi-peak fitting performed after subtraction of amorphous contribution. 


\section{A}
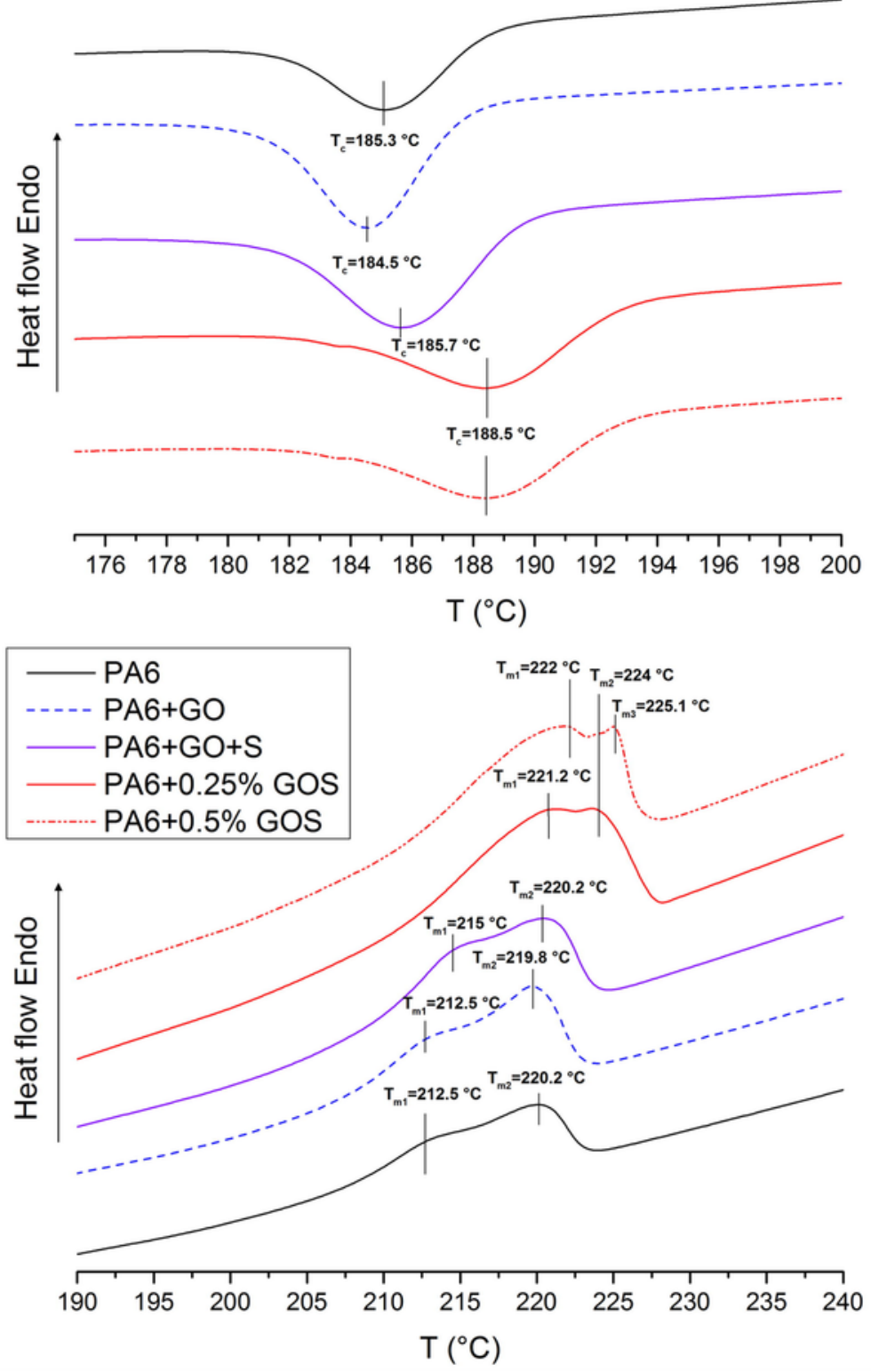

Fig. 10. DSC traces of nanocomposites: (A) cooling and (B) second heating scans. 


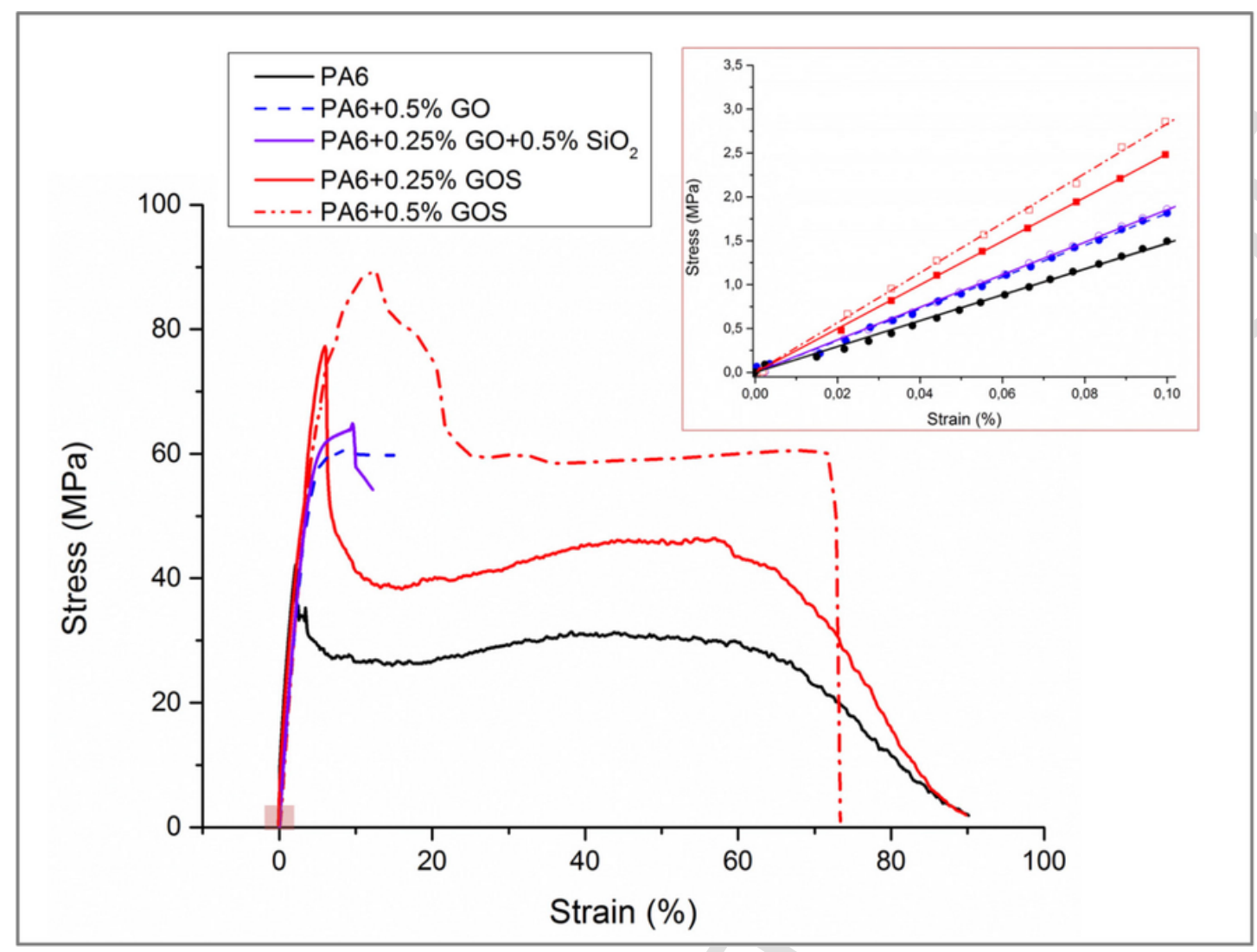

Fig. 11. Representative stress-strain curves of nanocomposites (inset: initial slope of each curves and linear fitting used to calculate E).

Table 2

Mechanical properties of nanocomposites.

\begin{tabular}{|c|c|c|c|c|c|}
\hline Sample & $\mathrm{E}[\mathrm{MPa}]$ & $\begin{array}{l}\mathrm{TS} \\
{[\mathrm{MPa}]}\end{array}$ & EB (\%) & $\begin{array}{l}\text { Toughness } \\
{\left[\mathrm{MJ} \mathrm{m}^{-3}\right]}\end{array}$ & $\begin{array}{l}\text { IS } \\
{\left[\mathrm{J} \mathrm{m}^{-1}\right]}\end{array}$ \\
\hline PA6 & $\begin{array}{c}1474 \\
( \pm 44)\end{array}$ & $42( \pm 4)$ & $\begin{array}{l}81 \\
( \pm 14)\end{array}$ & $22.51( \pm 3.9)$ & $\begin{array}{l}225 \\
( \pm 26)\end{array}$ \\
\hline $\mathrm{PA} 6+\mathrm{GO}$ & $\begin{array}{l}1813 \\
( \pm 91)\end{array}$ & $61( \pm 7)$ & $\begin{array}{l}15 \\
( \pm 3.2)\end{array}$ & $7.74( \pm 1.2)$ & $\begin{array}{l}154 \\
( \pm 37)\end{array}$ \\
\hline $\mathrm{PA} 6+\mathrm{GO}+\mathrm{S}$ & $\begin{array}{l}1854 \\
( \pm 64)\end{array}$ & $66( \pm 5)$ & $\begin{array}{l}16 \\
( \pm 2.9)\end{array}$ & $6.17( \pm 0.21)$ & $96( \pm 18)$ \\
\hline PA $6+0.25 \% \mathrm{GOS}$ & $\begin{array}{l}2401 \\
( \pm 81)\end{array}$ & $77( \pm 6)$ & $\begin{array}{l}60 \\
( \pm 11)\end{array}$ & $29.7( \pm 3.4)$ & \multirow{2}{*}{$\begin{array}{l}334 \\
( \pm 20) \\
388 \\
( \pm 22)\end{array}$} \\
\hline PA $6+0.5 \%$ GOS & $\begin{array}{l}2684 \\
( \pm 86)\end{array}$ & $88( \pm 8)$ & $\begin{array}{l}71 \\
( \pm 16)\end{array}$ & $48.2( \pm 4.2)$ & \\
\hline
\end{tabular}

\section{References}

[1] I. Rodriguez-Pastor, G. Ramos-Fernandez, H. Varela-Rizo, M. Terrones, I. Martin-Gullon, Towards the understanding of the graphene oxide structure: how to control the formation of humic-and fulvic-like oxidized debris, Carbon $\mathrm{N}$ Y. 84 (2015) 299-309, http://dx.doi.org/10.1016/j.carbon.2014.12.027.

[2] D.R. Dreyer, S. Park, C.W. Bielawski, R.S. Ruoff, The chemistry of graphene oxide, Chem. Soc. Rev. 39 (2010) 228-240, http://dx.doi.org/10.1039/ b917103g.

[3] X. Zhang, X. Fan, H. Li, C. Yan, Facile preparation route for graphene oxide reinforced polyamide 6 composites via in situ anionic ring-opening polymerization, J. Mater. Chem. 22 (2012) 24081, http://dx.doi.org/10.1039/c2jm34243j.

[4] X. Fu, C. Yao, G. Yang, Recent advances in graphene/polyamide 6 composites: a review, RSC Adv. 5 (2015) 61688-61702, http://dx.doi.org/10.1039/ C5RA09312K.

[5] A. Maio, R. Fucarino, R. Khatibi, S. Rosselli, M. Bruno, R. Scaffaro, A novel approach to prevent graphene oxide re-aggregation during the melt compound- ing with polymers, Compos. Sci. Technol. 119 (2015) 131-137, http://dx.doi. org/10.1016/j.compscitech.2015.10.006.

[6] E.L. Papadopoulou, F. Pignatelli, S. Marras, L. Marini, A. Davis, A. Athanassiou, et al., Nylon 6,6/graphene nanoplatelet composite films obtained from a new solvent, RSC Adv. 6 (2016) 6823-6831, http://dx.doi.org/10.1039/ c5ra23647a

[7] K. Scully, R. Bissessur, Decomposition kinetics of nylon-6/graphite and nylon-6/graphite oxide composites, Thermochim. Acta 490 (2009) 32-36, http:// dx.doi.org/10.1016/j.tca.2009.01.029.

[8] D. Zheng, G. Tang, H. Bin Zhang, Z.Z. Yu, F. Yavari, N. Koratkar, et al., In situ thermal reduction of graphene oxide for high electrical conductivity and low percolation threshold in polyamide 6 nanocomposites, Compos. Sci. Technol. 72 (2012) 284-289, http://dx.doi.org/10.1016/j.compscitech.2011.11.014.

[9] H.R. Pant, B. Pant, P. Pokharel, H.J. Kim, L.D. Tijing, C.H. Park, et al., Photocatalytic $\mathrm{TiO}_{2}-\mathrm{RGO} /$ nylon-6 spider-wave-like nano-nets via electrospinning and hydrothermal treatment, J. Memb. Sci. 429 (2013) 225-234, http://dx.doi.org/ 10.1016/j.memsci.2012.11.025.

[10] P. Ding, S. Su, N. Song, S. Tang, Y. Liu, L. Shi, Highly thermal conductive composites with polyamide- 6 covalently-grafted graphene by an in situ polymerization and thermal reduction process, Carbon N. Y. 66 (2014) 576-584, http://dx.doi.org/10.1016/j.carbon.2013.09.041.

[11] J. Tong, H.-X. Huang, M. Wu, Facile green fabrication of well dispersed poly(vinylidene fluoride)/graphene oxide nanocomposites with improved properties, Compos. Sci. Technol. 129 (2016) 183-190, http://dx.doi.org/10.1016/j compscitech.2016.04.027.

[12] A. Maio, S. Agnello, R. Khatibi, L. Botta, A. Alessi, A. Piazza, et al., A rapid and eco-friendly route to synthesize graphene-doped silica nanohybrids, J. Alloys Compd. 664 (2016) 428-438, http://dx.doi.org/10.1016/j.jallcom.2015.12. 137.

[13] A. Maio, R. Fucarino, R. Khatibi, L. Botta, S. Rosselli, M. Bruno, et al., Graphene oxide-silica nanohybrids as fillers for PA6 based nanocomposites, in: AIP Conf. Proc., American Institute of Physics Inc., 2014. pp. 438-441. doi: $10.1063 / 1.4876872$.

[14] N. Hong, L. Song, T.R. Hull, A.A. Stec, B. Wang, Y. Pan, et al., Facile preparation of graphene supported $\mathrm{Co}_{3} \mathrm{O}_{4}$ and $\mathrm{NiO}$ for reducing fire hazards of polyamide 6 composites, Mater. Chem. Phys. 142 (2013) 531-538, http://dx.doi. org/10.1016/j.matchemphys.2013.07.048 
[15] L.L. Li, S.H. Chen, W.J. Ma, Y.H. Cheng, Y.P. Tao, T.Z. Wu, et al., A novel reduced graphene oxide decorated with halloysite nanotubes (HNTs-d-rGO) hybrid composite and its flame-retardant application for polyamide 6, Express Polym. Lett. 8 (2014) 450-457, http://dx.doi.org/10.3144/expresspolymlett. 2014.48.

[16] R. Qian, J. Yu, C. Wu, X. Zhai, P. Jiang, Alumina-coated graphene sheet hybrids for electrically insulating polymer composites with high thermal conductivity, RSC Adv. 3 (2013) 17373-17379, http://dx.doi.org/10.1039/c3ra42104j.

[17] J. Ou, F. Wang, Y. Huang, D. Li, Y. Jiang, Q.-H. Qin, et al., Fabrication and cyto-compatibility of $\mathrm{Fe}_{3} \mathrm{O}_{4} / \mathrm{SiO}_{2} /$ graphene-CdTe QDs/CS nanocomposites for drug delivery, Colloid Surf. B Biointerf. 117 (2014) 466-472, http://dx.doi.org/ 10.1016/j.colsurfb.2013.12.003.

[18] N. Van Hoa, T.T. Khong, T. Thi Hoang Quyen, T. Si Trung, One-step facile synthesis of mesoporous graphene $/ \mathrm{Fe}_{3} \mathrm{O}_{4}$ /chitosan nanocomposite and its adsorption capacity for a textile dye, J. Water Process Eng. 9 (2016) 170-178, http://dx.doi.org/10.1016/j.jwpe.2015.12.005

[19] D. Peng, R.-P. Liang, H. Huang, J.-D. Qiu, Electrochemical immunosensor for carcinoembryonic antigen based on signal amplification strategy of graphene and $\mathrm{Fe}_{3} \mathrm{O}_{4} / \mathrm{Au}$ NPs, J. Electroanal. Chem. 761 (2016) 112-117, http://dx.doi. org/10.1016/j.jelechem.2015.12.014.

[20] B. Ramezanzadeh, Z. Haeri, M. Ramezanzadeh, A facile route of making silica nanoparticles-covered graphene oxide nanohybrids $\left(\mathrm{SiO}_{2}-\mathrm{GO}\right)$; fabrication of $\mathrm{SiO}_{2}-\mathrm{GO} /$ epoxy composite coating with superior barrier and corrosion protection performance, Chem. Eng. J. 303 (2016) 511-528, http://dx.doi.org/10.1016/ j.cej.2016.06.028

[21] D.C. Marcano, D.V. Kosynkin, J.M. Berlin, A. Sinitskii, Z. Sun, A. Slesarev, et al., Improved synthesis of graphene oxide, ACS Nano 4 (2010) 4806-4814, http://dx.doi.org/10.1021/nn1006368.

[22] A. Maio, D. Giallombardo, R. Scaffaro, A. Palumbo Piccionello, I. Pibiri, Synthesis of a fluorinated graphene oxide-silica nanohybrid: improving oxygen affinity, RSC Adv. 6 (2016) 46037-46047, http://dx.doi.org/10.1039/ c6ra02585d.

[23] R. Scaffaro, A. Maio, F. Lopresti, D. Giallombardo, L. Botta, M.L. Bondì, et al., Synthesis and self-assembly of a PEGylated-graphene aerogel, Compos. Sci. Technol. 128 (2016) 193-200, http://dx.doi.org/10.1016/j.compscitech.2016.03. 030 .

[24] A. Dimiev, D.V. Kosynkin, L.B. Alemany, P. Chaguine, J.M. Tour, Pristine graphite oxide, J. Am. Chem. Soc. 134 (2012) 2815-2822, http://dx.doi.org/10 1021/ja211531y

[25] T. Oh, Correlation between potential barrier and FTIR spectra in SiOC film with the $\mathrm{C}-\mathrm{O}$ bond of sp3 structure, Bull. Korean Chem. Soc. 30 (2009) 467-470. http://www.scopus.com/inward/record.url?eid=2-s2.0-84894897078\& partnerID $=$ tZOtx3y 1

[26] Y.L. Khung, S.H. Ngalim, L. Meda, D. Narducci, Preferential formation of Si-O-C over Si-C linkage upon thermal grafting on hydrogen-terminated silicon (1 11 1), Chemistry 20 (2014) 15151-15158, http://dx.doi.org/10.1002/chem. 201403014.

[27] A. Mathkar, T.N. Narayanan, L.B. Alemany, P. Cox, P. Nguyen, G. Gao, et al., Synthesis of fluorinated graphene oxide and its amphiphobic properties, Part. Part. Syst. Charact. 30 (2013) 266-272, http://dx.doi.org/10.1002/ppsc. 201200091.

[28] S. Agnello, A. Piazza, A. Alessi, A. Maio, R. Scaffaro, G. Buscarino, et al., Graphene oxide and Fumed silica graphene oxide nanocomposites modification by thermal treatments, in: NANOCON 2013 - Conf. Proceedings, 5th Int. Conf., TANGER Ltd., 2013. pp. 56-61. http://www.scopus.com/inward/record. url?eid=2-s2.0-84929398033\&partnerID=tZOtx3y1.
[29] S. Pan, I.A. Aksay, Factors controlling the size of graphene oxide sheets produced via the graphite oxide route, ACS Nano (2011) 4073-4083.

[30] E. Reynaud, T. Jouen, C. Gauthier, G. Vigier, J. Varlet, Nanofillers in polymeric matrix: a study on silica reinforced PA6, Polymer (Guildf) 42 (2001) 8759-8768, http://dx.doi.org/10.1016/S0032-3861(01)00446-3.

[31] H. Gu, Y. Guo, S.Y. Wong, C. He, X. Li, V.P.W. Shim, Effect of interphase and strain-rate on the tensile properties of polyamide 6 reinforced with functionalized silica nanoparticles, Compos. Sci. Technol. 75 (2013) 62-69, http://dx.doi. org/10.1016/j.compscitech.2012.12.004

[32] Y. Chen, F. Guo, A. Jachak, S.P. Kim, D. Datta, J. Liu, et al., Aerosol synthesis of cargo-filled graphene nanosacks, Nano Lett. 12 (2012) 1996-2002, http://dx. doi.org/10.1021/nl2045952.

[33] A. Maio, L. Botta, A.C. Tito, L. Pellegrino, M. Daghetta, R. Scaffaro, Statistical study of the influence of CNTs purification and plasma functionalization on the properties of polycarbonate-CNTs nanocomposites, Plasma Process. Polym. 11 (2014) 664-677, http://dx.doi.org/10.1002/ppap.201400008.

[34] M.T. Takemori, Towards an understanding of the heat distortion temperature of thermoplastics, Polym. Eng. Sci. 19 (1979) 1104-1109, http://dx.doi.org/10. 1002/pen.760191507.

[35] S. Spinella, G. Lo Re, B. Liu, J. Dorgan, Y. Habibi, P. Leclére, et al., Polylactide/cellulose nanocrystal nanocomposites: efficient routes for nanofiber modification and effects of nanofiber chemistry on PLA reinforcement, Polymer (United Kingdom) 65 (2015) 9-17, http://dx.doi.org/10.1016/j.polymer.2015.02. 048 .

[36] R. Scaffaro, A. Maio, A.C. Tito, High performance PA6/CNTs nanohybrid fibers prepared in the melt, Compos. Sci. Technol. 72 (2012) 1918-1923, http:// dx.doi.org/10.1016/j.compscitech.2012.08.010.

[37] Y. Wu, Y. Wang, H. Zhang, Y. Wang, Science and rubber - pristine clay nanocomposites prepared by co-coagulating rubber latex and clay aqueous suspension 65 (2005) 1195-1202, http://dx.doi.org/10.1016/j.compscitech.2004.11. 016.

[38] F. Meng, R. Zhao, Y. Zhan, X. Liu, Design of thorn-like micro/nanofibers: fabrication and controlled morphology for engineered composite materials applications, J. Mater. Chem. 21 (2011) 16385-16390, http://dx.doi.org/10.1039/ cljm12166a.

[39] F. Meng, Y. Zhan, R. Zhao, X. Liu, New strategy to reinforce and toughen composites via introducing thorns-like micro/nanofibers, Eur. Polym. J. 48 (2012) 74-78, http://dx.doi.org/10.1016/j.eurpolymj.2011.11.010.

[40] L. Petersson, K. Oksman, Science and biopolymer based nanocomposites: comparing layered silicates and microcrystalline cellulose as nanoreinforcement, Compos. Sci. Technol. 66 (2006) 2187-2196, http://dx.doi.org/10.1016/j. compscitech.2005.12.010.

[41] B.B.G. Uo, Ã.Q.Z. Ou, Y.L. Ei, D.J. Ia, Structure and performance of polyamide 6/halloysite nanotubes, Nanocomposites 41 (2009) 835-842, http:// dx.doi.org/10.1295/polymj.PJ2009110.

[42] R. Scaffaro, A. Maio, S. Agnello, A. Glisenti, Plasma functionalization of multiwalled carbon nanotubes and their use in the preparation of nylon 6-based nanohybrids, Plasma Process. Polym. 9 (2012) 503-512, http://dx.doi.org/10. 1002/ppap.201100140

[43] L. Gong, B. Yin, L.P. Li, M.B. Yang, Nylon-6/Graphene composites modified through polymeric modification of graphene, Compos. Part B Eng. 73 (2015) 49-56, http://dx.doi.org/10.1016/j.compositesb.2014.12.009.

[44] D. Dixon, P. Lemonine, J. Hamilton, G. Lubarsky, E. Archer, Graphene oxide-polyamide 6 nanocomposites produced via in situ polymerization, J. Thermoplast. Compos. Mater. 28 (2013) 372-389, http://dx.doi.org/10.1177/ 0892705713484749. 